\title{
Recurrent bone metastasis of follicular thyroid carcinoma with unknown primary site
}

\section{Ji Oh Mok', Chong Hwa Kim², Sung Rae Kim³ , Seung Hee Yü, and Kiyoung Lee}

${ }^{1}$ Division of Endocrinology and Metabolism, Department of Internal Medicine, Soonchunhyang University Bucheon Hospital

${ }^{2}$ Division of Endocrinology and Metabolism, Department of Internal Medicine, Sejong General Hospital

${ }^{3}$ Division of Endocrinology and Metabolism, Department of Internal Medicine, The Catholic University of Korea Bucheon ST.Mary's Hospital

${ }^{4}$ Division of Endocrinology and Metabolism, Department of Internal Medicine, Gachon University Gil Hospital, Incheon, South Korea

\section{INTRODUCTION}

Occurrence of follicular thyroid carcinoma (FTC) is limited almost within thyroid gland, but distant metastasis is discovered uncommonly as first presentation. We experienced a rare case about recurrent bone metastasis of FTC with unknown primary site.

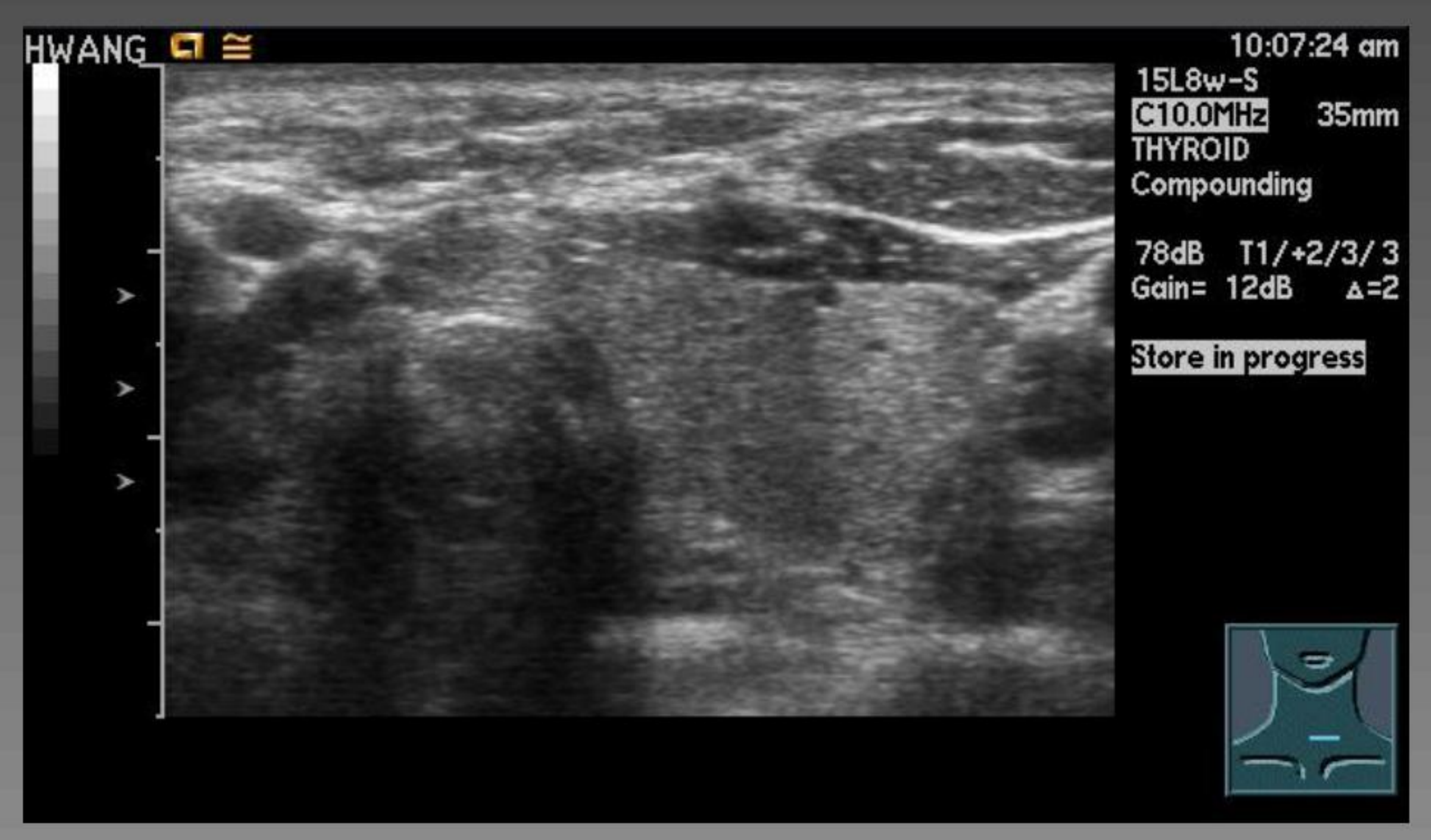

Figure 1. Thyroid ultrasonography showed no abnormal findings on left residual lobe.
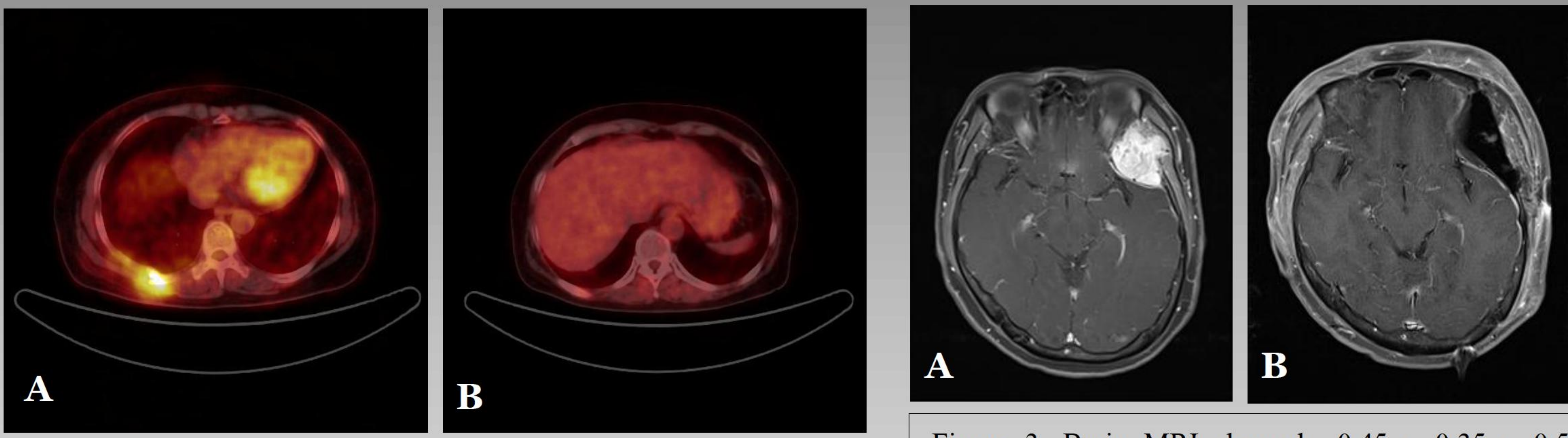

Figure 2. PET-CT showed increased uptake in the right 8 th rib before total thyroidectomy (A) and mild increased uptake in the posterior arc of right 9 th rib after 6 years $(B)$.

Figure 3. Brain MRI showed $0.45 \times 0.35 \times 0.52 \mathrm{~cm}$ heterogeneously enhancing mass in the left temporal bone (A) and no residual tumor in the tumornectomy site after surgery.

\section{CASE REPORT}

A 58-year-old woman complained about back pain for 3 years. A $5 \mathrm{~cm}$ sized mass in right 8 th posterior rib was discovered and performed resection. The result of pathology was bone metastasis of FTC. Thirty four years ago, she underwent right thyroidectomy because of benign nodule. Thyroid USG shows no specific findings on remained left lobe of thyroid gland, but PET-CT presents increased uptake in the left thyroid gland, bilateral hilar and mediastinal regions, and right 8th rib. Left thyroid gland was removed totally, but primary FTC wasn't discovered in left lobe. After operation, she received radioactive iodine (RAI) ablation with $100 \mathrm{mCi}$. However, recurrent bone metastasis of right 8 th posterior rib was found in PET-CT at 6 months after surgery. Repeated operation was performed for the lesion of right rib and pathological finding shows the same results as the first operation. Because of repeated bone metastasis and increased Tg level, she was ablated 4 times totally with $550 \mathrm{mCi}$ and underwent recurrent operations for bone metastasis of right rib. One year later, metastasis of left temporal bone was found newly and removed surgically with cranioplasty. The result of pathology was a metastasis of FTC such as the previous result. Currently, the patient is followed up in the outpatient department for conservative treatment.

\section{CONCLUSIONS}

With unknown primary site, recurrent bone metastasis of FTC is a rare case and should be treated with appropriate therapy because of poor prognosis. We report a case about recurrent bone metastasis of FTC managed with repeated operation and RAI ablation. 